Revue d'histoire de l'Amérique française

REYUE D.HISTOIRE DE L'AMÉRIQUE FRANÇAISE

\title{
Du lobbying pour la construction d'une infrastructure publique
}

\author{
Le système télégraphique dans le golfe du Saint-Laurent, \\ 1875-1895
}

\section{Éric Mauras}

Volume 60, numéro 3, hiver 2007

URI : https://id.erudit.org/iderudit/015962ar

DOI : https://doi.org/10.7202/015962ar

Aller au sommaire du numéro

\section{Éditeur(s)}

Institut d'histoire de l'Amérique française

ISSN

0035-2357 (imprimé)

1492-1383 (numérique)

Découvrir la revue

Citer cet article

Mauras, É. (2007). Du lobbying pour la construction d'une infrastructure publique : le système télégraphique dans le golfe du Saint-Laurent, 1875-1895. Revue d'histoire de l'Amérique française, 60(3), 325-354.

https://doi.org/10.7202/015962ar
Résumé de l'article

La notion de service public s'impose de plus en plus au Canada à partir du milieu du XIX ${ }^{\mathrm{e}}$ siècle, notamment en ce qui concerne les infrastructures urbaines. Nous proposons ici une étude sur l'action d'un groupe de pression, les marchands de Québec, auprès du gouvernement fédéral dans le but d'installer un réseau télégraphique dans le golfe du Saint-Laurent. Ce projet, présenté par ses promoteurs comme un service public, prend forme à partir de 1875. Il vise, en améliorant la sécurité de la navigation dans le golfe, à favoriser la place du port de Québec comme plaque tournante du trafic commercial canadien. Cet article retrace les actions du groupe de pression en faveur de leur projet, la mise en place de ce dernier par le gouvernement ainsi qu'une analyse économique de son fonctionnement entre 1880 et 1895 . Notre étude montre ainsi la distorsion existant entre l'efficacité pratiquement nulle du réseau télégraphique et l'image que s'en font les milieux d'affaires canadiens. Il s'agit d'un exemple rare d'une action de lobbying aux conséquences positives pour le Canada en général, mais négatives pour le groupe privé à l'origine du projet.
Tous droits réservés @ Institut d'histoire de l'Amérique française, 2007
Ce document est protégé par la loi sur le droit d'auteur. L’utilisation des services d'Érudit (y compris la reproduction) est assujettie à sa politique d'utilisation que vous pouvez consulter en ligne. 


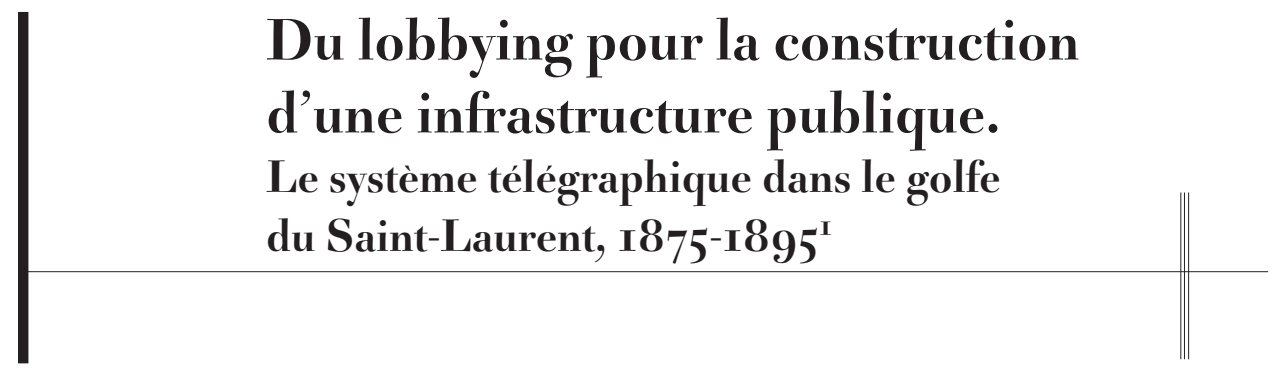

ÉRIC MaUras

Département d'histoire

Université de Montréal

RÉsumÉ • La notion de service public s'impose de plus en plus au Canada à partir du milieu du XIXe siècle, notamment en ce qui concerne les infrastructures urbaines. Nous proposons ici une étude sur l'action d'un groupe de pression, les marchands de Québec, auprès du gouvernement fédéral dans le but d'installer un réseau télégraphique dans le golfe du SaintLaurent. Ce projet, présenté par ses promoteurs comme un service public, prend forme à partir de 1875. II vise, en améliorant la sécurité de la navigation dans le golfe, à favoriser la place du port de Québec comme plaque tournante du trafic commercial canadien. Cet article retrace les actions du groupe de pression en faveur de leur projet, la mise en place de ce dernier par le gouvernement ainsi qu'une analyse économique de son fonctionnement entre 1880 et 1895. Notre étude montre ainsi la distorsion existant entre l'efficacité pratiquement nulle du réseau télégraphique et l'image que s'en font les milieux d'affaires canadiens. II s'agit d'un exemple rare d'une action de lobbying aux conséquences positives pour le Canada en général, mais négatives pour le groupe privé à l'origine du projet.

1. Cet article est issu d'un travail de recherche pour ma thèse de doctorat intitulée Entreprise, technologie et souveraineté. Les télécommunications au Canada, des origines à la Première Guerre mondiale et fait suite à une communication présentée en octobre 2005 à Shawinigan au congrès de l'Institut d'histoire de l'Amérique française. Je souhaite remercier particulièrement Michael Huberman, mon directeur, et Michèle Dagenais pour leurs judicieux conseils concernant cet article, Julie de Chantal et Karine Maurais pour leur relecture attentive de mon manuscrit, ainsi que les évaluateurs externes de la Revue d'histoire de l'Amérique française pour leurs commentaires pertinents et les suggestions apportées à la première version de ce texte. 
ABSTRACT - Public utilities became increasingly popular in Canada from the middle of the $19^{\text {th }}$ Century, especially with regards to urban infrastructures. This article examines the efforts of one pressure group, Quebec City merchants, to persuade the federal government to install a telegraph network in the Gulf of St. Lawrence. This project, which the promoters presented as a public service, began to take shape in 1875. By improving the security of shipping in the Gulf, it aimed to advance the position of the Port of Quebec as a hub for Canadian commercial traffic. The article traces the actions taken by the pressure group to promote their project, examines the government's installation of the network and provides an economic analysis of its operations between 1880 and 1895. It shows the discrepancy between the almost complete inefficiency of the telegraph network and the image that the Canadian business community had of it. This was a rare example of a lobbying effort whose consequences were positive for Canada as a whole, but negative for the private group behind the project.

«

Ce n'est que lorsqu'on a vue [sic] le danger de prêt [sic] que l'on peut apprécier à leur juste valeur les appareils qui vous ont fait éviter un désastre ${ }^{2}$." C'est par ces mots lourds de sens que Narcisse Rosa conclut, le 28 mars 1876, son témoignage devant les députés de la Chambre des communes. Cet armateur de Québec s'exprime en connaissance de cause. Six ans plus tôt, en 1870, un de ses navires endommagé dans le Bas-SaintLaurent avait dû jeter l'ancre dans une rade ouverte sur la côte gaspésienne. La nouvelle de l'accident avait mis trois jours à se rendre à Québec. En tout, il a fallu six jours pour voir arriver les secours sur le lieu de l'accident. Malheureusement, une violente tempête avait mis le navire en pièces entre-temps. L'équipage était sauf, mais le navire et sa cargaison, d'une valeur totale de 70000 \$, étaient perdus. Narcisse Rosa affirme «que s'il y’avait eu une ligne télégraphique sur la côte sud [...], j’aurais sauvé un de mes navires $[\ldots]$ avec son chargement ${ }^{3}$.»

Cette difficulté à obtenir des secours rapides est un problème fréquemment évoqué dans les récits de naufrage. Dans une lettre au Morning Chronicle, Pierre-Étienne Fortin, député de Gaspé, dénombre 119 accidents dans le golfe du Saint-Laurent entre 1870 et 1875 . Il soutient que pour une vingtaine d'entre eux, les pertes auraient pu être amoindries si des secours étaient parvenus plus rapidement sur les lieux du naufrage ${ }^{4}$. La réputation

2. «Rapport sur les avantages et la nécessité d'établir un réseau de télégraphe sous-marin dans le fleuve et golfe St. Laurent ", Journaux de la Chambre des communes du Canada, X (Session 1876): 66.

3. Id.

4. Pierre Fortin, «Système de télégraphe maritime pour le fleuve et le golfe St. Laurent», Morning Chronicle, 18 janvier 1876. 
du golfe du Saint-Laurent cause frayeur à plusieurs capitaines et équipages, notamment étrangers, parfois terrorisés au point de refuser de naviguer vers Québec et Montréal ${ }^{5}$. Les risques de la navigation sont fréquemment évoqués par les armateurs, d'où les pressions nombreuses exercées sur le gouvernement pour améliorer la signalisation et la communication sur le fleuve. Si les investissements sont conséquents pour les phares, bouées et sifflets d'alarme, la sécurité du golfe souffre d'un défaut majeur. Pendant l'hiver, les " sentinelles ${ }^{6}$ » du Saint-Laurent ne peuvent communiquer avec la terre ferme, ni avec les ports du golfe pour signifier les passages de navires et les éventuels accidents. Dans ce contexte, la solution la plus appropriée pour remédier à ce problème consiste en la construction de lignes télégraphiques aériennes et sous-marines.

Il s'agit ici d'un rare cas où le télégraphe est employé sans l'appui d'un chemin de fer. En effet, la plupart des lignes télégraphiques ont été mises en service en accord avec des compagnies ferroviaires, voire par elles directement ${ }^{7}$. Dans le cas du golfe du Saint-Laurent, les lignes télégraphiques doivent jouer un rôle similaire à celui qu'elles jouent pour les chemins de fer, c'est-à-dire rendre le transport plus sécuritaire. Les premières estimations de prix, ainsi que la difficulté à évaluer les conséquences directes d'un tel système, amènent les armateurs et les commerçants de Québec à rechercher le soutien de l'État pour leur projet.

Notre article vise à étudier la mise en place d'un service télégraphique dans le golfe du Saint-Laurent en tant que service public. Très peu de travaux historiques concernant l'industrie télégraphique canadienne s'intéressent à cette question précise. Parmi les travaux récents, Peter G. Cohen s'est penché sur la question de la transmission des informations au milieu du XIx ${ }^{\mathrm{e}}$ siècle en Amérique du Nord britannique, et particulièrement sur l'impact du télégraphe sur les journaux ${ }^{8}$. David R. Richeson et John Rowlandson ont étudié la mise en place du réseau télégraphique dans l'Ouest du Canada ${ }^{9}$. Les deux études s'intéressent particulièrement

5. "Rapport sur les avantages...", loc. cit., 65.

6. C'est ainsi que Pierre Fortin surnomme les gardiens de phares du golfe du Saint-Laurent.

7. Par exemple, la Canadian Pacific Telegraph Company, la Grand Trunk Telegraph Company ou la Canadian Northern Telegraph Company. La Montreal Telegraph Company a aussi signé des accords pour établir des lignes le long de l'Intercolonial Railway ou du Grand Trunk Railway.

8. Peter G. Cohen, "The Impact of the Telegraph on the Newspaper in the Mid-Nineteenth Century British North America», Urban Geography, 11,2 (1990): 107-129.

9. David R. Richeson, "The Telegraph and Community Formation in the North-West Territories» dans John E. Foster, dir., The Developing West: Essays on Canadian History in Honor of 
à la manière dont le télégraphe a influencé le développement de l'Ouest canadien tant sur le plan économique et administratif, pour le premier, que sur le plan socioculturel, pour le second. Précédemment, Andrew Robb s'était penché sur l'action individuelle en faveur du télégraphe du Canadien Pacifique et du projet Overland visant à relier l'Amérique à l'Europe, via la Sibérie ${ }^{10}$. Enfin, Shirley Maye Tillotson s'est intéressée aux questions salariales dans l'industrie télégraphique, notamment en ce qui a trait aux relations de genre et aux rapports hiérarchiques ${ }^{11}$.

D’un autre côté, plusieurs historiens ont étudié la mise en place des services publics dans la deuxième moitié du $\mathrm{XIX}^{\mathrm{e}}$ siècle, notamment Christopher Armstrong et Henri Vivian Nelles, dans leur importante synthèse sur les services publics urbains (tramway, électricité, gaz, téléphone...) au Canada ${ }^{12}$. La plupart des études historiques sur la mise en place des services publics concernent en premier lieu les infrastructures urbaines, par exemple l'approvisionnement en eau ${ }^{13}$, la mise en place d'un réseau téléphonique ${ }^{14}$ ou encore les relations industrielles en généra ${ }^{15}$. Pour les infrastructures de télécommunications, quelques travaux de synthèse sont disponibles, issus d'études d'économistes ou de spécialistes en communications ${ }^{16}$. Robert E. Babe et Gerald Michael McCabe ont

Lewis H. (Edmonton, University of Alberta Press, 1983), 137-153 ; John Rowlandson, Experiencing Space: A Socio-Cultural Case Study of the Canadian Government's Pioneer Telegraph Service in Western Canada, 1870-1904, mémoire de maîtrise (histoire), Université McGill, 1991.

10. Andrew Robb, «Edward Watkins and the Pacific Telegraph, 1861-1865 ", Ontario History, 65,4 (1973): 189-209; Andrew Robb, The Collins' Overland or Russian Extension Telegraph Project : A Pioneer Attempt to Establish Telegraphic Communications Between North America and Europe, mémoire de maîtrise (histoire), Simon Fraser University, 1968.

11. Shirley Maye Tillotson, Canadian Telegraphers, 1900-1930. A Case Study in Gender and Skill Hierarchies, mémoire de maîtrise (histoire), Queen’s University, 1988.

12. Christopher Armstrong et Henry Vivian Nelles, Monopoly's Moment. The Organization and Regulation of Canadian Utilities, 1830-1930 (Toronto, University of Toronto Press, 1988), $393 \mathrm{p}$.

13. Dany Fougères, L'approvisionnement en eau à Montréal. Du privé au public, 1796-1860 (Sillery, Septentrion, 2004), 472 p.

14. Claire Poitras, La cité au bout du fil. Le téléphone à Montréal de 1879 à 1930 (Montréal, Presses de l'Université de Montréal, 2000), 323 p.

15. Tom Traves, The State and Enterprise: Canadian Manufacturers and the Federal Government, 1917-1931 (Toronto, University of Toronto Press, 1979), 175 p. ; Paul Craven, "An Impartial Umpire": Industrial Relations and the Canadian State, 1900-1911 (Toronto, University of Toronto Press, 1980), 386 p.

16. Les principales recherches sur les relations entre État et entreprise privée dans le domaine des télécommunications canadiennes sont celles de Robert Steven Fortner, Messiahs and Monopolists. A Cultural History of Canadian Communications Systems, 1846-1914, thèse de doctorat (communication), Université de l'Illinois, 1978, et de Robert E. Babe, Telecommunication in Canada. Technology, Industry and Governement (Toronto, University of Toronto Press, 1990), 363 p. 
particulièrement étudié la régulation par l'État et l'instauration des services publics dans le domaine de la téléphonie ${ }^{17}$. Nous ne trouvons aucune synthèse de ce genre sur l'industrie télégraphique. Notre article se veut une contribution à l'ouverture de ce domaine de recherche.

Le télégraphe est, avec le chemin de fer, l'une des premières technologies à déployer un réseau à l'échelle du continent. Il importe de souligner que les entreprises privées qui exploitent cette technologie se trouvent souvent à rendre un service d'intérêt général auprès du public. Cela les amène ainsi à exiger le soutien de l'État afin de rendre leurs activités plus sécuritaires et prospères. Ce soutien se manifeste sous diverses formes, notamment par la mise en place de lois favorables, le versement de subventions ou, comme dans le cas qui nous concerne ici, par un investissement direct. De manière générale, à partir du milieu du XIx ${ }^{\mathrm{e}}$ siècle, l'État fédéral canadien intervient de plus en plus dans le domaine de la télégraphie.

Nous souhaitons dans cet article exposer le processus de formation de ce service public gouvernemental dans le golfe du Saint-Laurent. Plus spécifiquement, nous désirons montrer que sa mise en place résulte d'un croisement d'intérêts convergents de la part d'acteurs publics et privés. Premièrement, un groupe de pression, les marchands de Québec, essaye de pousser l'autorité publique à agir. Ensuite, les compagnies télégraphiques canadiennes laissent ostensiblement de côté une activité économique non rentable à leurs yeux. Enfin, l'État s'approprie cette activité comme symbole de la capacité d'action des milieux politiques sur la vie économique du pays.

Dans un premier temps, nous présenterons la situation initiale et la position du groupe de pression en 1875-1876. Nous préciserons les objectifs que le groupe s'est fixés et les actions entreprises pour y parvenir. Dans la seconde partie, nous suivrons pas à pas l'action du gouvernement de 1876 à 1896, ce qui nous permettra d'étudier la construction du réseau télégraphique. Enfin, nous dresserons un bilan de l'exploitation du réseau à partir des rapports annuels du ministère de la Marine et du ministère des Travaux publics. Nous évaluerons ainsi son impact économique et ses résultats finaux, tant pour la navigation marchande que pour les marchands de Québec et le gouvernement. Le choix de 1896 comme point

17. Robert E. Babe, "Control of Telephones. The Canadian Experience», Canadian Journal of Communication, 13 (1988): 16-29; Gerald Michael McCabe, Regulation of the Telephone Industry in Canada. The Formative Years, mémoire de maîtrise (sciences politiques), Université McGill, 1985. 
d'arrêt de notre article se justifie par la mise en service au tournant du siècle des premières stations de télégraphie sans fil, technologie pouvant biaiser une partie des données de notre étude.

\section{LES ACTIONS DU GROUPE DE PRESSION}

En 1875-1876, le port de Québec est le premier port de la côte atlantique canadienne sur le plan du tonnage, avec un trafic total de 1455638 tonneaux, et le troisième pour les mouvements de navires, avec 1854 arrivées et départs (derrière Halifax et Saint Jean) ${ }^{18}$. La navigation y est ouverte en général d'avril à novembre, soit huit mois par an. Néanmoins, la comparaison avec le port de New York lui est défavorable sous bien des aspects. Ce dernier est en effet libre de glace et nécessite un trajet de 25 miles seulement pour atteindre la haute mer, contre 585 miles pour Québec ${ }^{19}$. Enfin, la côte de la Nouvelle-Angleterre présente moins de difficultés et de dangers que la côte découpée du Saint-Laurent. L'action du groupe de pression a donc pour objectif de réduire l'impact de ces désavantages.

Pierre-Étienne Fortin est à l'origine des actions du groupe de pression. Cet homme a consacré une grande partie de sa carrière à défendre la cause de ses administrés en Gaspésie. Magistrat chargé de la promotion des pêcheries dans le golfe du Saint-Laurent de 1852 à 1867, il acquiert une excellente connaissance des conditions de navigation dans cette région. Membre du Parti conservateur, il est également député du comté de Gaspé à la Chambre des communes à Ottawa de 1867 à 1874 et de 1879 à 1889. Dans ses projets, Pierre-Étienne Fortin semble avoir agi de manière désintéressée. Sa seule volonté aurait été l'amélioration de la navigation dans le golfe et des conditions de vie des Gaspésiens. Son action comme magistrat lui aurait permis de gagner l'affection de ses compatriotes, affection qu'il leur rendait bien dans ses actions à Ottawa ${ }^{20}$. Très au fait des techniques de son époque, il agit activement dans le dossier de la construction d'un chemin de fer et d'une ligne télégraphique sur la côte nord de la Baie des Chaleurs, pour relier Bonaventure et Gaspé au chemin de fer de l'Intercolonial.

Au printemps de 1875 , six bateaux à vapeur de fort tonnage, emportant plus d'un millier de passagers sont signalés à l'entrée du golfe du Saint-

18. "Tableaux du commerce et de la navigation du Canada», Documents de la Session, X (1876): 704.

19. «Rapport sur les avantages...», loc. cit., 32.

20. Irène Bilas, «Fortin, Pierre-Étienne», Dictionnaire biographique du Canada (Québec/Toronto, Les Presses de l'Université Laval/University of Toronto Press), XI (1881-1890): 351-353. 
Laurent. Dix jours plus tard, aucun n'est parvenu à destination ${ }^{21}$. Cette nouvelle déclenche une vive émotion des deux côtés de l'Atlantique, à la fois chez les armateurs et les familles des passagers. Pierre-Étienne Fortin envoie alors une lettre à la rédaction du Canadien ${ }^{22}$. Il se montre rassurant et optimiste, précisant qu'à son avis, les navires sont simplement bloqués par les glaces, sans réel danger. Il ajoute qu'il serait parfaitement possible de recevoir des nouvelles si des câbles télégraphiques étaient immergés pour relier le Rocher-aux-Oiseaux au continent. Il achève son article par un résumé des avantages attendus d'une telle liaison, tant en termes de sécurité pour la navigation que de profits prévisibles pour une compagnie de télégraphe, sans pour autant s'avancer à ce sujet. Toutefois, Fortin semble sous-estimer le coût d'une telle ligne (longue de 98 miles, dont 62 immergés), puisqu'il prétend qu'elle ne coûterait que 40000 \$. Le projet est accueilli favorablement dans le pays, plusieurs journaux le diffusant avec bienveillance. Cet article, sans conséquences immédiates, expose néanmoins l'idée d'un réseau télégraphique de service public dans le golfe du Saint-Laurent.

Les marchands de Québec voient rapidement l'intérêt d'un tel système pour la navigation et les avantages qu'ils peuvent en retirer. Ils semblent également plus réalistes que Pierre-Étienne Fortin. Ayant probablement encore en mémoire l'aventure de la British North American Electric Telegraph Association ${ }^{23}$, ils estiment qu'un tel projet serait peu rentable pour une entreprise privée. En effet, le réseau, construit à l'avantage de tous, ne serait supporté et financé que par une poignée d'actionnaires. Le Board of Trade de Québec envisage de soumettre l'idée d'un tel système au Dominion Board of Trade lors d'une réunion à Ottawa en février 1876. Pierre-Étienne Fortin, l'ayant appris, se décide à envoyer une nouvelle lettre, directement au Board of Trade de Québec. Cette fois-ci, il présente un projet plus complet et plus développé, accompagné d'une carte ${ }^{24}$.

21. "Rapport sur les avantages...", loc. cit., 28.

22. La lettre de Fortin est publiée au complet dans le Morning Chronicle le 7 mai 1875 et dans le Canadien le lendemain.

23. La British North American Electric Telegraph Association, fondée en 1847 sur une proposition de Frederick Newton Gisborne, était principalement financée par des marchands de Québec. La compagnie souhaitait concurrencer la puissante Montreal Telegraph, mais sera rachetée par cette dernière en 1856, après plusieurs années d'échecs.

24. Cette lettre est aussi publiée en intégralité dans les pages du Morning Chronicle le 18 janvier 1876. 
Figure I

Projet de réseau télégraphique proposé par Pierre-Étienne Fortin en 1876 (lignes existantes en noir, lignes projetées en gris)

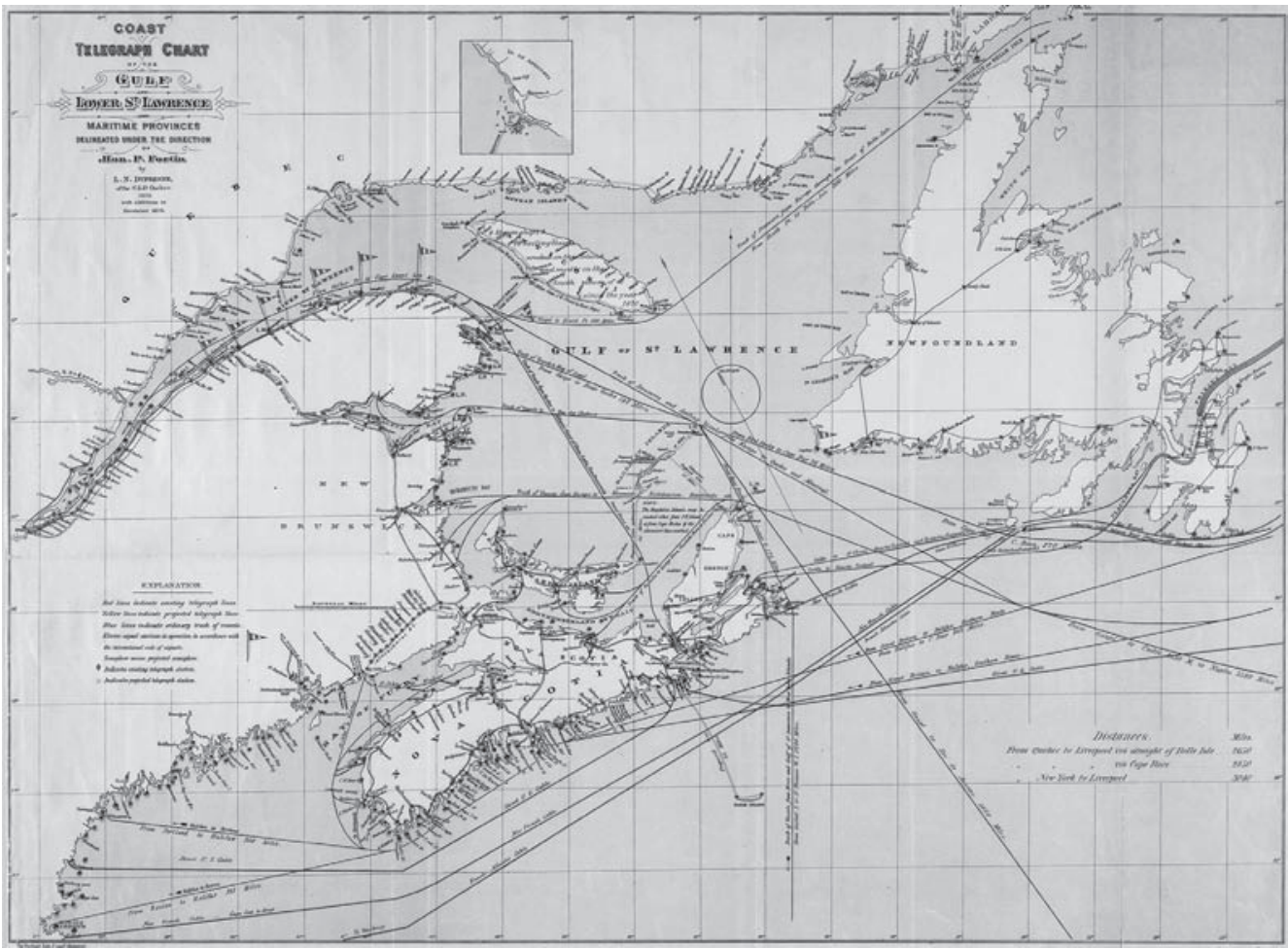

Référence: P.-É. Fortin, L.-N. Dufresne, Burland Lith. Co, "Coast Telegraph Chart of the Gulf and Lower St. Lawrence and Maritime Provinces», échelle 1:2 100 000, Montréal (Québec), 1879 (1876), 1 carte: noir \& blanc, 50,4 cm x 69,5 cm, Bibliothèque et Archives Canada, H2/1102/1879(1876), NMC 25305

Le projet élaboré par Pierre-Étienne Fortin prévoit la construction de sept lignes télégraphiques à travers le golfe (voir figure 1$)^{25}$. Ce système télégraphique serait complété par le service d'un vapeur, tenu prêt en tout temps et stationné à Gaspé. L'objectif de cet ambitieux projet est de rendre la navigation plus sécuritaire dans le golfe du Saint-Laurent en réduisant les délais des secours et, par conséquent, la gravité des nau-

25. BAC, NMC 25305, Coast telegraph chart of the Gulf and lower St. Lawrence and Maritime Provinces. Delineated under the direction of Hon. P. Fortin by L. N. Dufresne, after the C. L. O Quebec. 1876 with additions to December, 1879. The Burland Lith. Compy. Montreal. 
frages. Le Board of Trade de Québec estime que ce projet permettrait de pallier les désavantages du port de Québec et, dès lors, de rivaliser avec celui de New York. L'objectif en jeu est la primauté sur la côte atlantique, notamment par un accroissement de l'arrière-pays du port de Québec vers les Grands Lacs. Les marchands de Québec manquent peut-être d'argent, mais pas d'idées, et encore moins d'ambition, puisqu'ils semblent vouloir faire de Québec la plaque tournante du commerce canado-européen. Le Board of Trade de Québec reprend donc l'ensemble du projet à son compte pour le présenter à Ottawa et s'assurer du soutien du gouvernement. Le Dominion Board of Trade est un allié idéal pour obtenir ce soutien.

Nous disposons de peu d'indices pour évaluer l'action du Dominion Board of Trade. Celui-ci est sensible aux arguments avancés et partage les conclusions des armateurs et commerçants de Québec. Il est peu probable que le groupe de pression ait envisagé de financer ce projet par l'intermédiaire d'une entreprise privée sans prendre, au minimum, l'avis des administrateurs et techniciens de la plus grande compagnie télégraphique canadienne de l'époque, la Montreal Telegraph Company. Malheureusement, l'absence de traces de ce projet dans les archives de la compagnie pour les années 1875-1876 nous empêche de vérifier le sérieux des négociations à ce sujet ${ }^{26}$. L'Acte de l'Amérique du Nord britannique plaçant les lignes télégraphiques sous la responsabilité du gouvernement fédéral2 ${ }^{27}$, le groupe de pression va donc agir pour amener ce dernier à financer le réseau.

Le Dominion Board of Trade joue avec la notion de «l'avantage général du Canada». Sans s'attarder sur la question du financement, il insiste fortement sur les qualités du projet concernant l'ensemble de la navigation locale et internationale. Il précise que ce projet est essentiel à un "pays civilisé qui possède pareille étendue de côtes » et qu’il «contribuerait par là même à la prospérité du pays ${ }^{28}$ ». Les arguments employés sont en parfait accord avec la notion de service public, en émergence à cette époque au Canada. Le Dominion Board of Trade profite de l'occasion et active son réseau pour faire pression sur le gouvernement.

26. BAC, RG30, volume 10485, Montreal Telegraph Company, Minute Book $n^{\circ} 2$ (1857-1887). Le projet n'est mentionné nulle part dans les procès-verbaux du conseil d'administration de la compagnie. Néanmoins, il est probable que des discussions informelles se soient tenues entre la compagnie et le groupe de pression.

27. Acte de l'Amérique du Nord Britannique, 1867, Article 92, alinéa 10a.

28. "Rapport sur les avantages... », loc. cit., 57-60 et 67-68. 


\section{LA FORMATION D'UN COMITÉ PARLEMENTAIRE}

Depuis 1874, le gouvernement libéral de Mackenzie dirige les affaires du pays et n'est pas particulièrement enclin à appliquer une politique d'intervention économique trop poussée. Les manœuvres débutent le 16 février 1876 avec l'envoi d'une pétition de William Rae à la Chambre des communes, demandant «l'adoption de mesures à l'effet d'assurer l'extension du système télégraphique depuis l'île-du-Prince-Édouard jusqu'aux Iles de la Magdeleine [sic], et de là, jusqu'aux Rochers des Oiseaux, dans le centre du Golfe St Laurent ${ }^{29}$ ». Le 25 février, le Dominion Board of Trade met tout son prestige dans la bataille et envoie sa propre pétition. Jusqu'au 8 avril, douze pétitions sont envoyées à la Chambre des communes, provenant de chambres de commerce (Dominion, Québec et Lévis), de municipalités (Saint-Sauveur, Bellechasse, Percé, Gaspé - deux fois - et Lévis) et d'hommes d'affaires, dont le président de la Compagnie d'Assurance Maritime de l'Union ${ }^{30}$.

L'action du groupe de pression se concentre sur quelques députés de la Chambre. Deux députés sont particulièrement sensibles à ce projet. Il s'agit d'Hector Louis Langevin, député conservateur de Charlevoix, et de Théodore Robitaille, député conservateur de Bonaventure. À eux deux, ces députés forcent l'action de la Chambre des communes. Le 17 février 1876, le lendemain de l'envoi de la première pétition, Langevin interroge le gouvernement sur une éventuelle subvention à un projet télégraphique $^{31}$. Le 23 février, Jean-Baptiste Romuald Fiset, député libéral de Rimouski, apporte son soutien à Langevin et à Robitaille en interrogeant le gouvernement sur le projet de Pierre-Étienne Fortin ${ }^{32}$. Le 9 mars, à la suite des pétitions déjà déposées, Robitaille propose la formation d'un comité spécial pour "s'enquérir de la possibilité d'établir un système télégraphique sous-marin» dans le golfe du Saint-Laurent ${ }^{33}$. Après délibérations, la motion proposée par Robitaille est votée, créant ainsi un comité parlementaire de douze députés, soit cinq conservateurs, cinq libéraux et deux indépendants ${ }^{34}$. Parmi eux, cinq proviennent du Québec, trois de

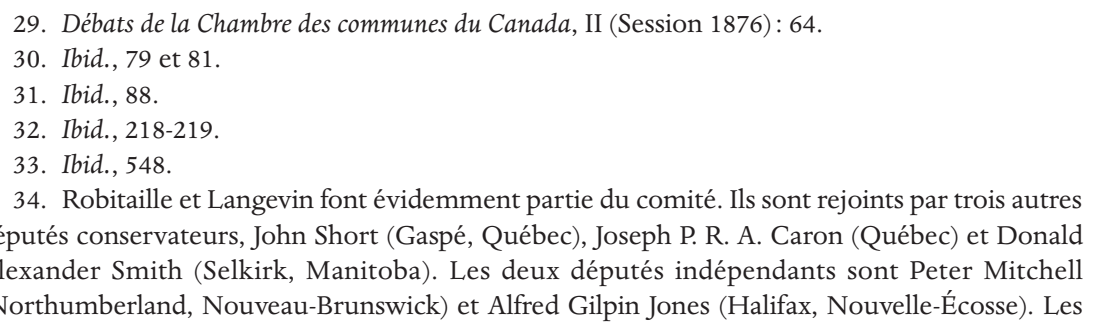


l'Ontario, deux de Nouvelle-Écosse, un du Manitoba et un du NouveauBrunwsick. De fait, huit députés sont directement intéressés par le projet.

Si le Dominion Board of Trade est bien à l'origine des actions des députés Langevin et Robitaille, ce choix est excellent. Robitaille est en quelque sorte l'alter ego de Pierre-Étienne Fortin. Député de Bonaventure, il a œuvré toute sa vie pour une meilleure intégration de la Gaspésie dans la Confédération canadienne ${ }^{35}$. Assurément, il perçoit ce projet comme essentiel pour le Canada et sa région. Quant à Langevin, il est le représentant par excellence des travaux publics. Si sa carrière politique a été plutôt houleuse, ses qualités d'ingénieur et d'organisateur sont indéniables $^{36}$. Son soutien au projet constitue un atout décisif. En effet, compte tenu de son expertise, les conclusions du comité ne sont pas remises en question.

Le comité siège à partir du 10 mars, pendant un mois. Vingt-cinq témoins sont entendus : trois sénateurs, cinq députés et cinq membres de l'administration témoignent devant les députés, dont William Smith, le ministre de la Marine et des Pêcheries, William F. Whitcher, le commissaire des pêcheries, et J. Tomlinson, le surintendant général des phares. Les douze autres témoignages proviennent d'armateurs, de représentants de compagnies de télégraphe et d'entrepreneurs. Tous les témoins reconnaissent les grandes qualités du projet. Le comité remet ses conclusions le 10 avril:

Votre comité a obtenu les témoignages de divers témoins, et [...] il est prêt à recommander, dans le but de satisfaire aux besoins du commerce et de la navigation, et de doter le fleuve et le golfe St. Laurent ainsi que les côtes de l'Atlantique de ce degré de sécurité indispensable à la protection des biens et des personnes, la nécessité d'établir un réseau complet de télégraphe sousmarin, reliant les îles du golfe et les endroits éloignés de la terre ferme aux lignes télégraphiques en existence dans le pays ${ }^{37}$.

libéraux sont William Hallet Ray (Annapolis, Nouvelle-Écosse), David Blain (York Ouest, Ontario), Thomas Workman (Montréal Ouest, Québec), Cyril Archibald (Stormont, Ontario) et John Charlton (Norfolk Nord, Ontario). Ibid., 554.

35. Marc Desjardins, "Robitaille, Théodore», Dictionnaire biographique du Canada (Québec/ Toronto, Les Presses de l’Université Laval/University of Toronto Press), XII (1891-1900): 994995.

36. Andrée Désilets, «Langevin, sir Hector Louis», Dictionnaire biographique du Canada (Québec/Toronto, Les Presses de l’Université Laval/University of Toronto Press), XIII (19011910): 617-622.

37. «Rapport sur les avantages...», loc. cit., 3. 
En définitive, le projet soutenu par le comité ressemble à peu de chose près à celui présenté en février par Pierre-Étienne Fortin. Point marquant, le gouvernement s'est donc tout simplement approprié un projet à l'origine privé, apparemment sans le modifier, ni le négocier. Les témoignages recueillis et présentés nous éclairent sur certains points.

Les propos des représentants de compagnies de télégraphe sont assez révélateurs. Ces hommes sont des ingénieurs compétents et tous reconnaissent l'utilité de ce réseau. Leurs évaluations financières servent de base aux estimations du comité, notamment le témoignage de James Paustie, surintendant du département des constructions de la Montreal Telegraph Company ${ }^{38}$. Pour sa part, Hugh Neilson, de la Dominion Telegraph Company, ne fournit que de vagues estimations, se retranchant derrière son ignorance de la géographie locale pour ne pas livrer de chiffres précis ${ }^{39}$. Malgré tout, le comité présente une estimation des dépenses prévisibles pour l'ensemble du réseau. Le projet consiste en trois divisions, qui seraient construites l'une après l'autre en deux à trois ans. La première division regroupe les lignes d'Anticosti et de la Rive-Nord du Saint-Laurent (137 250 \$). La seconde division comprend les îles-de-la-Madeleine, CapBreton, la pointe Miscou, la pointe Escouminac et les lignes de l'île-duPrince-Édouard (104 800 \$). La troisième division permet de compléter le réseau, notamment avec le Rocher-aux-Oiseaux, l'île Saint-Paul ainsi que des modifications pour l'île d'Anticosti (128 340\$). Cela représente un total de quatorze câbles sous-marins ou lignes terrestres à construire pour un investissement de l'ordre de 370390 \$ sur trois ans.

Les témoins-armateurs sont, quant à eux, enthousiastes. Nous avons déjà parlé de Narcisse Rosa, mais ses collègues sont aussi catégoriques que lui, tout comme les agents du gouvernement en charge de la marine ou des pêcheries. J. U. Gregory, agent du département de la Marine à Québec, voit le projet comme ayant «une importance vitale pour la marine marchande du Canada ${ }^{40}$ ». William Smith, ministre de la Marine, fournit une liste des naufrages dans le golfe de 1867 à 1875 . Il met aussi en lumière un point important, en précisant qu'un navire faisant naufrage avec sa cargaison constitue une perte douanière non négligeable pour le gouvernement fédéral ${ }^{41}$. W. F. Whitcher, commissaire aux pêcheries, s'intéresse plus particulièrement à l'emploi du télégraphe par les pêcheurs

\footnotetext{
38. Ibid., 9-10.

39. Ibid., 5-6.

40. Ibid., 12-13.

41. Ibid., 20.
} 
et les gains prévisibles pour eux ${ }^{42}$. Pour leur part, les députés et sénateurs entendus sont tous en faveur du projet, qu'ils jugent unanimement vital pour leurs circonscriptions. A. Murray, du Bureau de l'Assurance de Montréal, précise, quant à lui, quelques taux d'assurance pour la navigation maritime dans le golfe et les compare à ceux de New York ${ }^{43}$. Enfin, le témoignage de Pierre-Étienne Fortin est probablement le plus long et le plus exhaustif, puisqu'il s'intéresse à tous les détails du projet et résume les arguments en faveur du réseau télégraphique ${ }^{44}$.

L'objectif visé est de rendre la navigation sécuritaire dans le golfe, en réduisant la gravité des naufrages. Les bénéfices attendus sont la baisse des taux d'assurance maritime et l'accroissement du trafic dans le golfe. Le comité, par les témoignages recueillis, résume très bien les ambitions des marchands de Québec. Reste à passer des paroles aux actes.

\section{LA MISE EN PLACE DU RÉSEAU TÉLÉGRAPHIQUE}

Le moins que l'on puisse en dire, c'est que ce passage est laborieux. Il est vrai que la période se prête mal aux investissements massifs, puisqu'une crise économique secoue le pays. Nous l'avons vu, le rapport précisait que le réseau pouvait être installé en deux ou trois ans environ. Mais si les députés Robitaille et Langevin ont pu forcer l'action de la Chambre des communes, ils ne peuvent faire de même avec le gouvernement. Avec le recul, il semblerait même que le gouvernement Mackenzie ait cédé sur la question du comité pour mieux enterrer le rapport ensuite. Après sa publication, il n'est plus fait mention de ce projet sous le gouvernement Mackenzie. On note cependant un investissement en 1877 pour construire la ligne de la Rive-Sud, de Matane à Rivière-au-Renard, et ceinturer ainsi la Gaspésie. Des discussions avaient été engagées dès 1876 et seront poursuivies pour la construction de la ligne en $1878^{45}$. À la session 1877 , l'attribution d'une subvention totale de 15000 \$ est votée par le parlement, à la Montreal Telegraph Company pour l'établissement de cette ligne ${ }^{46}$.

Ce n'est qu'après le retour au pouvoir de Macdonald en 1878 que les choses évoluent. Tout juste après les élections, le Dominion Board of Trade organise une conférence à Ottawa et y invite Pierre-Étienne Fortin,
42. Ibid., 57-60.
43. Ibid., 60-61.
44. Ibid., 27-57.
45. BAC, RG30, volume 10485, Montreal Telegraph Company, Minute Book nº 2 (1857-1887), 157, 171 et 183 .
46. "Subsides», Actes du Parlement de la puissance du Canada, 40 Victoria, chapitre 1, 1877. 
récemment réélu député à la Chambre des communes. Cette conférence est un succès, permettant de présenter le projet et d'en faire l'éloge, en reprenant les grandes lignes du rapport parlementaire de $1876^{47}$. De leur côté, Langevin et Robitaille n'ont pas oublié ce rapport et, selon toute vraisemblance, le rappellent à Macdonald, dont le gouvernement met progressivement en place la Politique nationale. Si le chemin de fer en a été le volet le plus marquant, le réseau télégraphique du Saint-Laurent répond à la même logique. Il s'agit de financer un outil qui favorise l'essor économique du pays. Le pays étant toujours en pleine crise économique, le gouvernement essaie de minimiser ses dépenses. Il inscrit donc dans le budget de 1879 une proposition de subvention annuelle pour la mise en place du réseau, tel qu'il a été présenté dans le rapport de 1876. La subvention annuelle s'élève à $15000 \$$, pour payer l'entretien du réseau $(3000$ \$) et les intérêts d'un emprunt de 300000 \$ à $4 \%(12000$ \$) qui serait souscrit par la compagnie privée en charge de la construction du réseau télégraphique ${ }^{48}$. La subvention fait l'objet d'une loi spéciale, votée le 15 mai $1879^{49}$. La proposition est immédiatement envoyée aux quatre principales compagnies privées au Canada, c'est-à-dire la Montreal Telegraph Company, la Dominion Telegraph Company, la Western Union Telegraph Company et la New York, Newfoundland and London Telegraph Company ${ }^{50}$.

L'échec est total. Aucune compagnie ne répond. La Montreal Telegraph Company ne daigne même pas discuter de cette proposition au cours de ses conseils d'administration ${ }^{51}$. En fait, cette proposition ne satisfait ni le groupe de pression ni les compagnies privées. Pour eux, ce projet doit être pris en charge intégralement par le gouvernement fédéral. Le mutisme de toutes les compagnies est le coup de grâce. Après avoir proclamé et vanté les mérites de ce réseau à l'avantage de tous les Canadiens, le gouvernement ne peut plus faire marche arrière. Il est donc contraint d'agir lui-même.

47. Bureau de commerce de Montréal et de Québec, Système télégraphique pour les côtes et les $\hat{\imath} l e s d u$ golfe et $d u$ bas du fleuve St. Laurent et les côtes des provinces maritimes dans ses rapports avec la marine, les pêcheries et le service des signaux (Montréal, La Minerve, 1879), 19-44.

48. Débats de la Chambre des communes du Canada, VII (Session 1879): 1684.

49. "Acte à l'effet d'accorder une subvention annuelle pour aider à la construction et à l'entretien de communications télégraphiques avec les îles d'Anticosti et de la Madeleine», Actes du Parlement de la puissance du Canada, 42 Victoria, chapitre 5, 1879.

50. BAC, RG11, volume 2992, Estimates of Canada For the Financial Year Ending $30^{\text {th }}$ June 1880 , $79-80$.

51. BAC, RG30, volume 10485, Montreal Telegraph Company, Minute Book $n^{\circ} 2$ (1857-1887). 
Figure 2

État du réseau télégraphique gouvernemental dans le golfe du Saint-Laurent en 1898

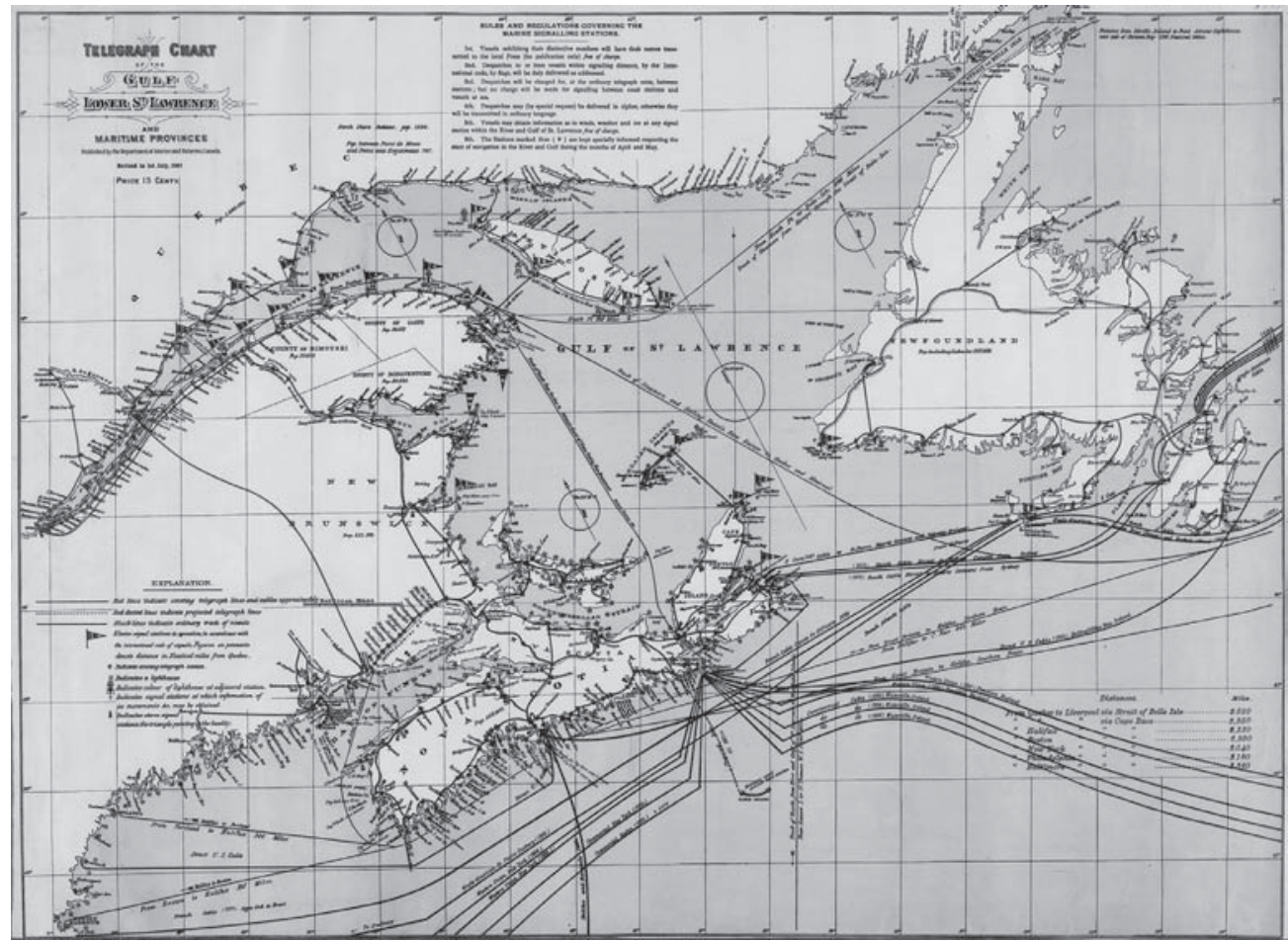

Référence : Canada, Department of Marine and Fisheries, «Telegraph Chart of the Gulf and Lower St. Lawrence and Maritime Provinces», échelle 1:2 100 000, Montréal (Québec), 1898, 1 carte : noir \& blanc, 50,4 $\mathrm{cm} \mathrm{x} \mathrm{69,1} \mathrm{cm,} \mathrm{Bibliothèque} \mathrm{et} \mathrm{Archives} \mathrm{Canada,} \mathrm{H2/1102/1898,}$ NMC 25308

Le 7 mai 1880, le gouvernement fait abroger la loi de subvention votée à la session de $1879^{52}$. Il dégage ensuite des fonds pour financer le réseau, capitalisant $10000 \$$ de la subvention prévue à hauteur de $5 \%$. Cela permet d'amasser une somme de $200000 \$$ pour la session $1880-1881$. Le gouvernement ne fait pas les choses à moitié. Alors que le comité de 1876 prévoyait un délai de construction de deux à trois ans, ce laps de temps est ramené à un an. Par un juste retour des choses, Langevin, ayant repris son poste de ministre des Travaux publics le 20 mai 1879, est responsable de l'établissement du réseau télégraphique.

52. "Acte à l'effet d'abroger l'acte quarante-deux Victoria, chapitre cinq, accordant une subvention annuelle pour aider à certaines communications télégraphiques ", Actes du Parlement de la puissance du Canada, 43 Victoria, c.14, 1880. 
D'importants travaux débutent à l'automne de 1880 (voir figure 2) (53 $^{53}$. Les lignes terrestres sur Anticosti et les Îles-de-la-Madeleine sont construites entre septembre et début novembre. Trois câbles sous-marins sont posés en octobre 1880, reliant l'île d'Anticosti, le Rocher-aux-Oiseaux et les Îlesde-la-Madeleine. La ligne de l'île du Cap-Breton, de Sydney à Meat Cove, est achevée en juin $1881^{54}$. Enfin, la ligne de la Rive-Nord est commencée, entre Murray Bay (aujourd'hui La Malbaie) et Mille-Vaches (aujourd'hui Longue-Rive) en traversant le Saguenay entre août 1880 et novembre 1881. Un embranchement allant de Murray Bay à Chicoutimi est aussi mis en service en septembre $1881^{55}$. Pour soutenir cet effort, les Bureaux de commerce de Québec et Montréal envoient deux pétitions au gouvernement en mai 1881 , l'enjoignant de poursuivre la construction du réseau ${ }^{56}$. Les plans sont d'ailleurs modifiés régulièrement. Les lignes de Chatham à Escouminac $^{57}$ et de Mabou à Chéticamp ${ }^{58}$, sur l'île de Cap Breton, et de la pointe Miscou sont ajoutées au projet entre 1884 et 1892. Enfin, les derniers câbles sont posés en 1890-1891: celui reliant Mingan à l'île d'Anticosti et celui entre Cap-Breton et l'île Saint-Paul'59. Par contre, les lignes prévues sur l'île-du-Prince-Édouard ne seront jamais construites.

\section{L'EXPLOITATION DU RÉSEAU ET SES RÉSULTATS FINANCIERS}

Les marchands de Québec et du Dominion Board of Trade ont atteint leur objectif, la construction du réseau télégraphique et sa gestion aux frais de l'État, après trois ans d'action. Sur le plan financier, le réseau télégraphique représente une sérieuse dépense pour le gouvernement fédéral (voir figure 3). En nous basant sur les crédits votés et dépensés annuellement par le gouvernement ${ }^{60}$, nous notons que le réseau s'est construit en trois étapes distinctes. La mise en place de l'armature centrale en constitue la première. Elle se déroule, comme nous l'avons vu, en

53. BAC, NMC 25308, Telegraph chart of the Gulf and lower St. Lawrence and Maritime Provinces. Published by the Department of Marine and Fisheries, Canada. Revised to $1^{\text {st }} J u l y, 1898$.

54. "Rapport général du ministère des Travaux Publics», Documents de la Session, XIII (1880) et XIV (1881).

55. Id.

56. Bureau de commerce de Montréal et de Québec, Système télégraphique..., op. cit., 45-46.

57. «Rapport général du ministère des Travaux Publics», Documents de la Session, XVIII (1885).

58. "Rapport général du ministère des Travaux Publics», Documents de la Session, XX (1887).

59. "Rapport général du ministère des Travaux Publics», Documents de la Session, XXIV (1891) et XXV (1892).

60. "Subsides", Actes du Parlement de la puissance du Canada, 38 Victoria à 58 Victoria (1875 à 1895). 
Figure 3

Crédits votés et dépenses de construction annuelles du gouvernement fédéral pour les années I880-|88| à |894-1895.

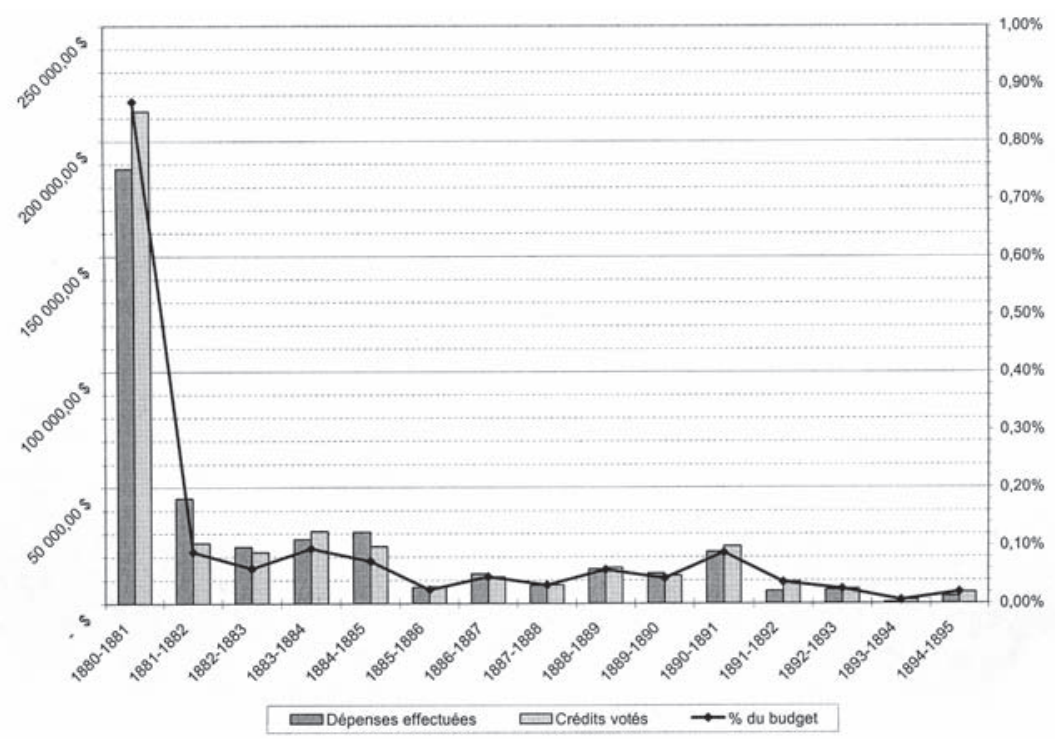

1880-1881. L'investissement de cette année-là représente à lui seul près de $50 \%$ de l'investissement global sur les quinze années étudiées ${ }^{61}$. Nous ne retrouvons pas un tel investissement dans un réseau télégraphique avant la session 1899-1900 pour la construction de la ligne du Yukon ${ }^{62}$.

La seconde étape s'étend sur quatre ans, de 1881 à 1885 , et totalise environ $25 \%$ de l'investissement total. Il s'agit de terminer l'armature du réseau pour le rendre aussi performant que possible. La dernière étape, de 1886 à 1896, vise à compléter les zones moins importantes et laissées de côté lors de l'investissement initial. Comme nous pouvons le voir, la somme totale dépensée est de $417429,70 \$$ sur 15 ans. Le réseau a pris une ampleur considérable depuis la proposition de Pierre-Étienne Fortin en 1875. L'estimation de $40000 \$$ avancée à l'époque était très optimiste, les câbles du Rocher-aux-Oiseaux et des Îles-de-la-Madeleine ayant coûté en réalité prés de 91250 \$.

Les dépenses d'entretien pour le réseau sont elles aussi importantes (voir figure 4). Nous ne pouvons savoir s'il s'agit de la conséquence d'économies réalisées sur la qualité des lignes construites ou plutôt de l'envi-

61. «Rapport général du ministère des Travaux Publics», Documents de la session, XIII (1880) et XIV (1881).

62. «Subsides», Actes du Parlement du Canada, 62 Victoria (1899) et 63 Victoria (1900). 
ronnement naturel exigeant et souvent sauvage de la région. Toujours est-il que les dépenses annuelles de fonctionnement sont, en moyenne, de l'ordre de $21329,74 \$, 56 \%$ de ce montant concernant les réparations des lignes et des câbles sous-marins ${ }^{63}$. La dépense pour l'entretien était estimée, dans la subvention de 1879 , à un total de $3000 \$$ par an en moyenne. Le gouvernement faisait donc preuve d'un incroyable optimisme. Ces dépenses servent principalement à entretenir les câbles sousmarins, souvent rompus. Le câble d'Anticosti a ainsi été endommagé à trois reprises, en avril 1887, durant l'hiver $1888-1889$ et au printemps $1891^{64}$. Le câble des Îles-de-la-Madeleine a été encore moins fiable puisqu’il fut endommagé à six reprises, créant chaque fois une coupure totale des communications ${ }^{65}$. De plus, la plupart des coupures ont lieu au printemps (quatre fois en quinze ans), moment critique pour la navigation dans le golfe du Saint-Laurent. Mais la palme de la non-fiabilité revient au câble du Rocher-aux-Oiseaux, coupé huit fois en dix ans. Il ne fonctionne convenablement qu'aux printemps 1884 et 1885 . Compte tenu de ce problème récurrent, le ministère des Travaux publics décide tout simplement de l'abandonner en décembre 1889 , au moment de son ultime panne. À l'automne 1890, le câble est relevé, réparé et immergé de nouveau pour relier l'île Saint-Paul à Cap-Breton. Et il ne sera pas plus fiable pour autant, puisqu'il sera coupé à deux reprises entre 1891 et $1895^{66}$. Ce manque de fiabilité aura un impact important sur l'efficacité et l'aide du réseau pour la navigation dans le Saint-Laurent au cours des années suivantes.

À ces dépenses d'entretien, il faut ajouter les frais de fonctionnement du service des signaux. En effet, les dépêches télégraphiques transitant entre les différents tronçons du réseau du gouvernement doivent passer par des lignes privées. Le service des signaux dispose donc d'un budget pour relier les vingt-neuf stations mises en place dans le golfe. En fait, seules seize stations sont reliées directement au réseau du gouvernement ${ }^{67}$. Treize d'entre elles sont reliées par le biais des lignes de la Great

63. Id.

64. "Rapport général du ministère des Travaux Publics», Documents de la session, XX à XXIV (1887 à 1891).

65. Les coupures sont signalées au printemps 1883, 1884 et 1886, à l'été 1888 , au printemps 1894 et à l'automne 1895. "Rapport général du ministère des Travaux Publics », Documents de la session, XIV à XXVIII (1881 à 1895).

66. "Rapport général du ministère des travaux Publics», Documents de la session, XXIV à XXVIII (1891 à 1895).

67. Il s'agit des stations de la rive nord du Saint-Laurent (4), d'Escouminac, d'Anticosti (4), de la Madeleine (3), du Rocher-aux-Oiseaux, de Cap-Breton (2) et du Cap Ray. «Rapport général du ministère des Travaux Publics», Documents de la session, XXII (1889) et XXIII (1890). 
Figure 4

Dépenses annuelles d'entretien (maintenance et salaires) et service des signaux pour les années I88I-I882 à 1894-1895



North Western Telegraph Company ${ }^{68}$. La dépense moyenne annuelle pour le service des signaux a été de l'ordre de 8790,27 \$. Au total, le gouvernement paie donc 30120 \$ par an en moyenne pour le bon fonctionnement du réseau télégraphique, soit près de dix fois la somme envisagée en 1879.

Néanmoins, ce réseau n'est pas non plus un gouffre sans fond, puisqu'il rapporte aussi un peu au gouvernement (voir figure 5). Lorsqu'une compagnie privée, un armateur ou un assureur veut communiquer avec un navire, il lui faut payer un télégramme au gouvernement. Malgré des tarifs prohibitifs ${ }^{69}$, celui-ci a quand même réussi à engranger une moyenne de $5373,85 \$$ par an ${ }^{70}$. Il reste néanmoins un déficit annuel moyen de l'ordre de 25000 \$. En évaluant l'ensemble des dépenses de construction et d'entretien et les revenus générés, le réseau du Saint-Laurent a coûté un total

68. Il s'agit des stations de la rive sud du Saint-Laurent (11) et de Gaspésie (2). La Great North Western Telegraph Company loue et exploite les lignes de la Montréal Telegraph Company depuis 1881. Id.

69. Le tarif est de l'ordre de $1,05 \$$ pour 10 mots et de 6 cents supplémentaires par mot, contre une moyenne de 25 cents pour dix mots et de 1 cent par mot supplémentaire sur les lignes de la Great North Western Telegraph Company. Id.

70. "Rapport général du ministère des travaux Publics», Documents de la session, XIV à XXVIII (1891 à 1895). 
Figure 5

Recettes et dépenses annuelles des lignes télégraphiques dans le golfe du Saint-Laurent pour les années I88I-1882 à 1894-1895

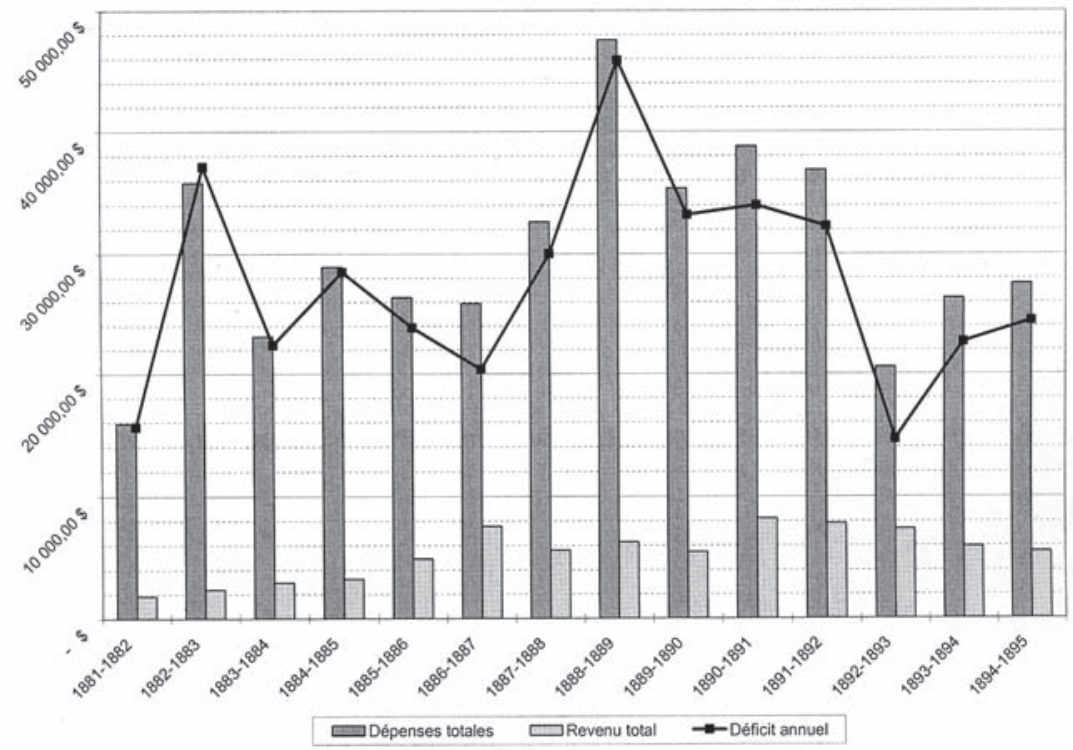

de 754624,28 \$ au gouvernement fédéral, soit un coût annuel moyen de $53901,73 \$$. À titre de comparaison, le réseau télégraphique de la Montreal Telegraph Company avait rapporté, pour la seule année 1880, 550840 \$ et coûté $358676 \$$, soit un bénéfice de $192164 \$$. Et ce réseau couvrait près de 12703 miles de lignes ${ }^{71}$, alors que le gouvernement fédéral gérait un réseau de 1387 miles seulement. La comparaison est plutôt défavorable ${ }^{72}$. Sur le plan financier, le réseau constitue donc une dépense sérieuse pour le gouvernement fédéral.

Cette analyse nous permet de vérifier la pertinence de l'action entreprise par le groupe de pression. Ce dernier a choisi de faire bâtir et exploiter ce réseau par l'autorité publique en se basant sur des hypothèses et des projections financières. Quinze ans plus tard, il est clair que ce choix était le bon, financièrement parlant du moins. En effet, cette apparente réussite financière est à relativiser par rapport au service public fourni aux navigateurs et armateurs du Saint-Laurent.

71. BAC, RG30, volume 10485, Montreal Telegraph Company, Minute Book no 2 (1857-1887).

72. La comparaison entre les gestions publiques et privées dans le domaine de la télégraphie serait probablement très instructive. 


\section{L'IMPACT DU RÉSEAU TÉLÉGRAPHIQUE SUR LA NAVIGATION}

Si les marchands de Québec ont atteint leur objectif initial en faisant financer et gérer le réseau par le gouvernement fédéral, il ne s'agit là que d'un début, les marchands ayant des visées plus larges. Leur objectif réel est la réduction des coûts maritimes par l'amélioration de la sécurité dans le golfe et l'accroissement de leurs revenus par l'augmentation du trafic pour le port de Québec. Pour étudier l’impact du réseau télégraphique sur la navigation, nous avons compilé les naufrages enregistrés entre 1875 et 1895 , pour les régions du golfe du Saint-Laurent, de la baie de Fundy et de la côte atlantique de la Nouvelle-Écosse ${ }^{73}$.

Nous avons séparé les trois zones géographiques pour obtenir des groupes témoins. En effet, la baie de Fundy n'a qu'un équipement télégraphique sommaire, mis en service en 1881. La côte atlantique de la Nouvelle-Écosse, pour sa part, ne dispose d'aucun équipement télégraphique gouvernemental et d'aucune station de signalisation. Quelques lignes privées existent, mais leur impact sur la navigation semble très marginal. Nous avons relevé la nature de chaque accident enregistré, le lieu et les dégâts estimés. D’autres informations, comme le type de navire et son tonnage, ont aussi été utilisées. Nous avons également relevé le trafic maritime en direction de l'Atlantique pour tous les ports canadiens pour les années 1875 à $1895^{74}$. Nous n'avons compté que le trafic au long cours, c'est-à-dire les navires venant ou allant à la mer. Le cabotage et les navires de pêche ne sont pas comptabilisés dans le calcul. Comme la plupart des accidents concernent des navires de haute mer, les statistiques établies sont, à notre avis, fiables. De même, nous n'avons comptabilisé que les accidents ayant eu lieu près des côtes canadiennes et lorsque le navire allait ou venait d'un port canadien. Au vu des témoignages lors d'une enquête en $1890^{75}$, nous avions supposé que le réseau télégraphique avait eu un impact important sur la navigation, en réduisant la gravité des naufrages, atteignant ainsi l'objectif visé par les marchands de Québec. Il n'en est rien.

Tout d'abord, les chiffres bruts semblent indiquer une réelle efficacité du réseau télégraphique dans le golfe du Saint-Laurent (voir tableau 1).

73 «Rapport annuel du département de la Marine», Documents de la session, VIII à XXVIII (1875 à 1895).

74. Id.

75. «Rapport général du ministère des travaux publics», Documents de la session, XXIV (1891). 
Entre 1875-1880 et 1890-1895, le nombre d'accidents annuels dans le golfe diminue de près de $60 \%$. La comparaison avec les autres zones est favorable puisque la côte atlantique voit sa moyenne annuelle diminuer de $33 \%$ et que la baie de Fundy, au contraire, voit sa moyenne s'accroître de $30 \%$. En ce qui concerne le tonnage, les chiffres sont similaires (voir tableau 2). Le golfe a vu ses pertes (partielles ou totales) annuelles moyennes chuter de près de $70 \%$. La côte atlantique suit la même courbe avec une diminution de $42 \%$. Quant à la baie de Fundy, sa moyenne annuelle affiche une parfaite stabilité, malgré l'augmentation du nombre d'accidents.

Tableau I

Moyenne annuelle des navires accidentés (dont pertes totales)

\begin{tabular}{l|c|c|c|c}
\hline & $\mathbf{1 8 7 5 - 1 8 8 0}$ & $\mathbf{1 8 8 0 - 1 8 8 5}$ & $\mathbf{1 8 8 5 - 1 8 9 0}$ & $\mathbf{1 8 9 0 - 1 8 9 5}$ \\
\hline Golfe du Saint-Laurent & $63(25)$ & $44(18)$ & $28(11)$ & $25(11)$ \\
\hline Côte Atlantique & $49(25)$ & $49(24)$ & $42(18)$ & $34(14)$ \\
\hline Baie de Fundy & $22(9)$ & $20(9)$ & $25(10)$ & $29(8)$ \\
\hline
\end{tabular}

Tableau 2

Moyenne annuelle du tonnage accidenté (en tonneaux)

\begin{tabular}{l|c|c|c|c}
\hline & $\mathbf{1 8 7 5 - 1 8 8 0}$ & $\mathbf{1 8 8 0 - 1 8 8 5}$ & $\mathbf{1 8 8 5 - 1 8 9 0}$ & $\mathbf{1 8 9 0 - 1 8 9 5}$ \\
\hline Golfe du Saint-Laurent & 24444 & 19004 & 11715 & 7337 \\
\hline Côte Atlantique & 11067 & 12641 & 10011 & 6518 \\
\hline Baie de Fundy & 5519 & 5605 & 5040 & 5578 \\
\hline
\end{tabular}

Au premier regard, le réseau télégraphique aurait donc permis une baisse plus importante du nombre d'accidents dans le Saint-Laurent. Pour affiner cette analyse, nous avons calculé un indice permettant de mesurer la dangerosité des côtes pour les trois zones géographiques étudiées, en comparant le nombre d'accidents par rapport au trafic circulant le long de ces côtes (voir tableau 3). Les résultats sont très différents des chiffres bruts. Le golfe voit son indice de dangerosité diminuer presque de moitié (45\%) entre 1875-1880 et 1880-1895. Il reste néanmoins la zone la plus dangereuse des trois. Comparativement, la côte atlantique voit le danger s'amenuiser de manière encore plus flagrante, avec un indice chutant de près de $63 \%$. La palme revient à la baie de Fundy avec une moyenne très basse qui se réduit encore plus avec une baisse de $28 \%$. Cette analyse permet donc de pondérer les chiffres bruts. En effet, la chute apparemment importante du nombre d'accidents dans le golfe du Saint-Laurent 
est relative, puisque le taux d'accident ne diminue que de moitié, alors qu'il baisse encore plus fortement pour la côte atlantique, qui n'est pas équipée en télégraphe par le gouvernement, ni en station de signalisation. Enfin, la baisse importante du nombre d'accidents dans le golfe s'explique aussi par la diminution du trafic en nombre de navires, cela découlant d'un accroissement important de la taille des navires circulant dans le golfe.

\section{Tableau 3}

Pourcentage annuel moyen d'accidents par rapport au trafic (nombre d'accidents/nombre de navires en transit)

\begin{tabular}{l|c|c|c|c}
\hline & $\mathbf{1 8 7 5 - 1 8 8 0}$ & $\mathbf{1 8 8 0 - 1 8 8 5}$ & $\mathbf{1 8 8 5 - 1 8 9 0}$ & $\mathbf{1 8 9 0 - 1 8 9 5}$ \\
\hline Golfe du Saint-Laurent & 13,38 & 10,84 & 7,20 & 7,31 \\
\hline Côte Atlantique & 9,28 & 7,31 & 5,14 & 3,46 \\
\hline Baie de Fundy & 3,37 & 2,26 & 1,87 & 2,42 \\
\hline
\end{tabular}

Tableau 4

Pourcentage annuel moyen de navires perdus par rapport aux accidents (nombre de navires perdus/nombre de navires accidentés)

\begin{tabular}{l|c|c|c|c}
\hline & $\mathbf{1 8 7 5 - 1 8 8 0}$ & $\mathbf{1 8 8 0 - 1 8 8 5}$ & $\mathbf{1 8 8 5 - 1 8 9 0}$ & $\mathbf{1 8 9 0 - 1 8 9 5}$ \\
\hline Golfe du Saint-Laurent & 40 & 41,1 & 38,13 & 43,65 \\
\hline Côte Atlantique & 51,64 & 48,18 & 42,31 & 41,67 \\
\hline Baie de Fundy & 42,73 & 45,10 & 40,80 & 28,28 \\
\hline
\end{tabular}

L'efficacité du réseau télégraphique est donc difficile à établir clairement. Cela devient encore plus difficile lorsque nous nous penchons sur la gravité des naufrages. En effet, de l'avis unanime des armateurs et marchands de Québec, le télégraphe joue un rôle important lorsqu'il est capable de prévenir rapidement les secours d'un accident, permettant ainsi d'éviter la perte totale d'un navire accidenté ou échoué. Si le télégraphe a vraiment joué un rôle majeur dans la diminution du nombre d'accidents dans le golfe du Saint-Laurent, la diminution de la gravité des naufrages devrait en être un corollaire. Nous avons donc isolé les accidents indiquant une perte partielle de ceux indiquant la perte totale du navire et de sa cargaison (voir tableau 4). Le résultat est inattendu.

En effet, contrairement aux affirmations et aux témoignages de l'époque, la gravité des naufrages dans le golfe augmente avec l'installation du télégraphe. Cela signifie qu'un navire accidenté dans le golfe a presque deux chances sur cinq d'être une perte totale, quel que soit l'équi- 
pement en télégraphe. La côte atlantique, non équipée, voit par contre la gravité de ses naufrages diminuer. Enfin, la baie de Fundy confirme sa réputation de sécurité, avec une très forte diminution de la gravité des accidents. Il est donc possible de conclure que le télégraphe n'a pas joué le rôle escompté, car son action ne se reflète absolument pas dans la gravité des naufrages, contrairement aux affirmations de Pierre-Étienne Fortin en 1876.

Ces chiffres sont confirmés lorsque nous étudions plus précisément les régions de l'île d'Anticosti et des Îles-de-la-Madeleine, réputées pour leur dangerosité. En compilant les accidents entre 1875 et 1895, nous avons séparé ceux ayant eu lieu lorsque le service télégraphique était installé et opérationnel, des accidents survenus lorsque le service n'existait pas ou, quand il existait, lorsque les câbles étaient endommagés ou coupés. Pour les Îles-de-la-Madeleine, la gravité des naufrages est de 51,79\% lorsque le service n'existait pas, et de $48 \%$ lorsqu'il existait. Pour l'île d'Anticosti, le taux de perte totale augmente après l'installation des lignes télégraphiques, avec un bond de $56 \%$ (sans le service) à $70 \%$ (avec le service). Tout cela nous confirme l'idée que le réseau télégraphique est loin d'avoir eu l'impact escompté sur la navigation.

Malgré tout, les témoignages recueillis en 1890 louent l'efficacité du réseau télégraphique. Ainsi, les taux d'assurance ont chuté pour la navigation vers Québec et Montréal. Le rapport de 1890 signale que la chute des tarifs depuis 1880 est de l'ordre de $50 \%$ (on avait estimé la baisse maximale à $25 \%$ en $\left.1876^{76}\right)$. En fait, si les chiffres bruts montrent une efficacité apparente, le calcul du danger des côtes et de la gravité des naufrages laisse penser que la baisse du nombre des accidents dans le golfe n’est pas réellement due au télégraphe. Dans ce cas, pourquoi les témoignages sont-ils aussi élogieux?

Les chiffres nous indiquent, en 1875-1880, que la côte atlantique est potentiellement aussi dangereuse que le golfe du Saint-Laurent. Un navire circulant dans le golfe a plus de chances d'avoir un accident mais, dans ce cas, il s'en sortira plus souvent que sur la côte atlantique.

La terrible réputation du Saint-Laurent semble donc surestimée. Par contre, les récits de naufrages tragiques dans le Saint-Laurent abondent. Sans doute la population a-t-elle, inconsciemment, considéré les naufrages catastrophiques dans le golfe comme étant la norme habituelle. Un naufrage particulièrement terrible, dont les survivants auraient péri ou sur- 
vécu dans d'atroces conditions, sera diffusé dans plusieurs journaux, sans manquer de préciser qu'il s'agit d'un exemple des naufrages de la région. À partir de là, la population, et notamment les marins et armateurs, peut s'imaginer une région redoutable, de sinistre réputation.

Là où le télégraphe joue un rôle, c'est justement en supprimant ces terribles récits de naufrages. Voyant moins de reportages de ce type dans les journaux après la mise en service du système télégraphique, la population peut croire que ce réseau a permis d'améliorer grandement la sécurité de la navigation. Même si ce n'est pas le cas, en éliminant les naufrages les plus extrêmes, le télégraphe donne l'illusion d'une efficacité redoutable, alors que les faits semblent prouver qu'il n'a pratiquement eu aucun impact sur les moyennes annuelles. C'est de là que provient l'apparence d'efficacité du télégraphe, expliquant ainsi les témoignages élogieux et la baisse des tarifs d'assurance. Une recherche plus détaillée sur le rôle des journaux dans la diffusion des récits de naufrages serait sûrement instructive à ce sujet ${ }^{77}$.

Il ne faut pas non plus négliger les autres facteurs pouvant expliquer la baisse des accidents sur la côte est du Canada. Par exemple, dans les années 1880-1890, nous notons un accroissement de la proportion des navires à vapeur par rapport aux voiliers. De même, les premières législations sur les certificats de capitaine et de second favorisent un accroissement dans la qualité et l'expérience des équipages naviguant dans le golfe. Enfin, le gouvernement continue d'améliorer la signalisation sur le Saint-Laurent (phares, bouées, sifflets...). Il semblerait, en définitive, que le réseau télégraphique ne soit qu'un outil parmi un ensemble de mesures mises en place par le gouvernement dans le but de rendre la navigation du Saint-Laurent plus sécuritaire.

Reste la dernière question, à savoir le gain des marchands de Québec. Le verdict est sans appel: l'installation du réseau télégraphique n'a aucun effet bénéfique sur l'activité du port de Québec. Nous avons comparé les quatre principaux ports de l'est du Canada, à savoir Québec, Montréal, Halifax et Saint-Jean (voir tableau 5). Dans l'ensemble, le tonnage annuel moyen circulant a augmenté de $25 \%$ pour les quatre ports. Le taux le plus élevé a été celui de Montréal (+ 111\%), suivi par Halifax (+ 49\%) et SaintJean $(+29 \%)$. Québec, pour sa part, a perdu un tiers de son trafic. En

77. Cet aspect m’a été suggéré à la suite de la conférence de France Normand, «Les représentations de l'espace maritime à travers les récits de naufrages (1850-1900): ce que nous disent les tribunaux d'enquêtes maritimes ", $58^{\mathrm{e}}$ congrès annuel de l'Institut d'histoire de l'Amérique française, tenu à Shawinigan les 21 et 22 octobre 2005. 
1875-1876, le port de Québec pouvait se glorifier d'être le premier port canadien concernant le tonnage, avec 1455638 tonneaux (pour 1854 mouvements de navires). En 1894-1895, il est en quatrième place avec 900728 tonneaux (-39\%) et seulement 598 mouvements de navires.

Tableau 5

Trafic annuel moyen en tonneaux par port (base $100=1875-1880$ )

\begin{tabular}{lc|c|c|c}
\hline & $\mathbf{1 8 7 5 - 1 8 8 0}$ & $\mathbf{1 8 8 0 - 1 8 8 5}$ & $\mathbf{1 8 8 5 - 1 8 9 0}$ & $\mathbf{1 8 9 0 - 1 8 9 5}$ \\
\hline Québec & $1453559(100)$ & $1402459(98)$ & $1073825(75)$ & $957920(67)$ \\
\hline Montréal & $703330(100)$ & $921942(131)$ & $1146585(163)$ & $1486540(211)$ \\
\hline Halifax & $862115(100)$ & $1128967(131)$ & $1196554(139)$ & $1283075(149)$ \\
\hline Saint-Jean & $836986(100)$ & $953459(114)$ & $1006937(120)$ & $1075597(129)$ \\
\hline
\end{tabular}

Même si les raisons de ce déclin sont complexes, il est clair que le télégraphe n'a pas permis de l'enrayer ${ }^{78}$. En fait, si le projet a été mis en œuvre par les marchands de Québec, il est aussi soutenu par les marchands de Montréal ${ }^{79}$. Durant la période étudiée, nous pouvons constater une légère hausse du trafic pour les deux ports, de l'ordre de $15 \%$ entre 1875 et $1895^{80}$. Cette légère hausse cache en réalité un transfert de trafic important de Québec vers Montréal entre 1880 et 1890. Montréal dépasse Québec pour le trafic en 1886 et pour les mouvements de navires en 1890 .

\section{CONCLUSION}

L'étude de l'action de lobbying pour la construction et l'entretien d'un réseau de communication télégraphique dans le golfe du Saint-Laurent est intéressante sous plusieurs aspects. Tout d'abord, elle permet de comprendre l'action d'un groupe de pression sur l'autorité publique. Les marchands de Québec utilisent diverses stratégies pour parvenir à l'objectif souhaité. Ils agissent directement à travers l'envoi de pétitions et indirectement à travers leurs relations avec le Dominion Board of Trade et avec quelques députés de la Chambre des communes. Ils montent aussi une campagne de relations publiques, en utilisant notamment le charisme de Pierre-Étienne Fortin lors d'une conférence publique organisée spéciale-

78. Pour une étude plus complète sur la situation du port de Québec entre 1875 et 1895, voir France Normand, Naviguer le Saint Laurent à la fin du XIX siècle. Une étude de la batellerie du port de Québec (Sainte-Foy, Les Presses de l'Université Laval, 1997), 283 p.

79. Bureau de commerce de Montréal et de Québec, Système télégraphique..., op. cit., 45-46.

80. "Rapport annuel du département de la marine», Documents de la session, VIII à XXVIII (1875 à 1895). 
ment pour l'occasion et en publiant ses lettres dans la presse de Québec. Dans cet exercice de communication, le groupe de pression joue habilement avec la notion de service public alors en émergence au Canada afin d'obtenir le soutien de l'État dans une entreprise dont il espère tirer profit.

Ensuite, nous avons mis en lumière l'activité des entreprises privées au sujet de ce projet. En tant qu'industriels, les dirigeants de ces compagnies sont conscients des implications économiques et financières qu'entraînerait la mise en place d'un tel réseau. Chaque compagnie possédant des réseaux télégraphiques dans la région se retrouve dans une situation inconfortable. En effet, à la fin du XIx ${ }^{\mathrm{e}}$ siècle, le télégraphe est une technologie synonyme de progrès, notamment depuis l'établissement des premières liaisons transatlantiques dans les années 1865-1866. Dans un monde industriel et innovateur, les compagnies doivent conserver une certaine image de prestige auprès du public. Une campagne importante favorisant un projet présenté "à l'avantage général du Canada», mais qu'elles savent potentiellement déficitaire, est une situation pour le moins embarrassante. En faisant la sourde oreille aux appels du gouvernement à ce sujet, elles exercent donc une pression sur ce dernier pour qu'il réalise lui-même le projet. Leur but ici est de laisser un projet, vu comme non rentable, aux mains de l'autorité publique, aussi longtemps qu'il ne concurrence pas les lignes privées.

Ensuite, nous avons étudié la réponse du gouvernement canadien aux pressions exercées à son encontre. Le moins que l'on puisse dire est qu'il oppose une résistance symbolique. Dans un premier temps, les actions entreprises semblent porter leurs fruits puisqu'un comité parlementaire est formé pour s'occuper de ce projet. Néanmoins, le gouvernement libéral de Mackenzie, fidèle à son idéologie économique, refuse de donner suite aux conclusions de ce comité. Par contre, le gouvernement conservateur de Macdonald cède presque immédiatement. Les arguments du groupe de pression concordent avec les arguments en faveur de la Politique nationale. Nous avons ici un excellent exemple d'action de la Politique nationale en dehors du domaine du chemin de fer et de la colonisation de l'Ouest. Ce qui est valable pour justifier l'investissement dans le Canadian Pacific Railway l'est aussi pour justifier le service de télégraphe dans le Saint-Laurent. De plus, le gouvernement gère déjà un service télégraphique en Colombie-Britannique depuis $1871^{81}$.

81. N'ayant pas trouvé de repreneur, le gouvernement fédéral a pris à sa charge les lignes du gouvernement de Colombie-Britannique lors de l'entrée de la province dans la Confédération en 1871. 
De fait, l'action du groupe de pression a été couronnée de succès, autant par les arguments avancés que par la faible résistance du gouvernement. Il est même possible d'envisager l'hypothèse que celui-ci ait utilisé le groupe de pression comme prétexte pour s'autoriser à agir dans un domaine économique jusqu'alors entièrement aux mains des compagnies privées.

Néanmoins, comme nous l'avons démontré, l'impact du réseau télégraphique tient davantage dans l'image que se fait le public de son efficacité. Néanmoins, cette apparence d'efficacité est un résultat non négligeable. D’après les statistiques compilées des naufrages, les pertes financières annuelles moyennes dans le golfe auraient diminué de près de 260000 \$ en 15 ans (voir tableau 6). En comparant ce chiffre avec ceux des autres régions maritimes, nous pouvons constater que cette baisse est bien plus importante qu'ailleurs. Par rapport au coût annuel moyen du réseau pour le gouvernement (53 901,73\$), nous croyons que le réseau télégraphique a pu être «rentable» pour la navigation. Nous n'avons pas pu quantifier exactement son rôle dans ce domaine, étant donné les nombreuses mesures gouvernementales ayant eu un impact sur la navigation entre 1875 et 1895 . Nous pensons, néanmoins, que son efficacité, bien que discutable en réalité, a favorisé l'économie canadienne en général. N’oublions pas non plus que le réseau télégraphique sert aussi à transmettre les bulletins des pêcheries. Nous formulons l'hypothèse que cela a entraîné une hausse des activités halieutiques dans le golfe du SaintLaurent et, par le fait même, une hausse des revenus pour les pêcheurs du golfe ${ }^{82}$.

Tableau 6

Pertes financières annuelles moyennes (en dollars)

\begin{tabular}{l|c|c|c|c}
\hline & $\mathbf{1 8 7 5 - 1 8 8 0}$ & $\mathbf{1 8 8 0 - 1 8 8 5}$ & $\mathbf{1 8 8 5 - 1 8 9 0}$ & $\mathbf{1 8 9 0 - 1 8 9 5}$ \\
\hline Golfe du Saint-Laurent & 478986 & 390110 & 191805 & 219815 \\
\hline Côte Atlantique & 196379 & 367702 & 131832 & 86719 \\
\hline Baie de Fundy & 71025 & 76948 & 103547 & 63189 \\
\hline
\end{tabular}

Enfin, point essentiel pour les navigateurs, les gains en capital, notamment sur les assurances et les frais de navigation, sont importants, même s'ils ne sont que la conséquence d'une vision biaisée de l'efficacité réelle

82. Cette hypothèse serait évidemment à vérifier par une analyse détaillée des conditions des pêcheurs dans le golfe et sur la côte atlantique du Canada, notamment les revenus, les capitaux investis et la productivité. 
du réseau. Le télégraphe justifie ici son statut de technologie qui, par un investissement minime, produit une importante économie en capital ${ }^{83}$. Malgré les apparences défavorables et le déficit important dû à cet investissement, le gouvernement fédéral a su exploiter ce réseau au sein d'un ensemble de mesures avantageuses pour l'économie du Canada en général. Le gain économique est donc appréciable bien que limité. Néanmoins, si ce réseau télégraphique a eu des conséquences positives, c'est plus grâce à son efficacité supposée que réelle.

Il s'agit donc d'un excellent exemple de mise en ouvre d'un changement institutionnel sous l'action d'un groupe social dans le but de s'approprier un gain économique. Cet exemple s'inscrit dans le cadre plus large de l'émergence des services publics au cours des années 1870-1890. La mise en place du réseau télégraphique dans le golfe du Saint-Laurent est en réalité un compromis entre les intérêts publics et privés. Le gouvernement fédéral, malgré la dépense, a des raisons d'être satisfait de la situation. Son réseau semble favoriser la croissance économique du pays. De plus, il a mis les pieds dans une industrie jusqu'alors dirigée uniquement par des intérêts privés. Cette expérience servira quelques années plus tard, notamment lors de la pose de lignes télégraphiques au Yukon ou pour le câble du Pacifique.

Les entreprises privées ont aussi des raisons de se réjouir. Leur image publique reste intacte et elles ont évité de se plonger dans un gouffre financier sans espoir de gains financiers immédiats. L’existence de lignes télégraphiques gouvernementales est un désagrément limité tant que ces dernières ne concurrencent pas les lignes privées sur les territoires les plus rentables.

Par contre, pour les marchands de Québec, le résultat est plus douteux. Le réseau fonctionne et semble efficace, ce que la réalité dément. Les taux d'assurance et les frais de navigation diminuent néanmoins. Dans le projet initial des marchands, le réseau télégraphique du Saint-Laurent devait permettre d'accroître la sécurité de la navigation entre Québec et l'océan Atlantique, c'est-à-dire vers l'Europe. L'outil télégraphique était donc envisagé par le Board of Trade de Québec comme un outil pour concurrencer New York, devenir le premier port de l'Atlantique et la plaque tournante du trafic commercial canadien. Malheureusement, cet outil était aussi utile aux marchands de Montréal. En ce qui concerne la liaison

83. Alexander J. Field, "The Magnetic Telegraph, Price and Quantity Data and the New Management of Capital», Journal of Economic History, 52 (juin 1992): 401-413. 
avec l'Ouest du Canada, les marchands de Québec ont échoué à obtenir une prolongation de la ligne du Canadien Pacifique jusqu'à leur port. En captant le trafic en provenance de l'Ouest et en profitant de la sécurité améliorée dans le golfe du Saint-Laurent, les marchands de Montréal sont les réels bénéficiaires de cette action de lobbying. Comme nous l'avons vu, à partir des années 1880-1890, le port de Québec perd de son importance et ne peut plus lutter avec Montréal, principalement en raison de sa position géographique dans le réseau ferroviaire canadien. Au final, c'est Montréal qui devient la plaque tournante du trafic commercial entre le Canada et l'Europe.

En conclusion, notre article permet de présenter un exemple d'une action de lobbying aux conséquences positives pour la population et le Canada en général, mais négatives pour le groupe privé à l'origine du projet. Ce genre de résultat mérite d'être mis en valeur, particulièrement pour la fin du xix ${ }^{\mathrm{e}}$ siècle. Dans la lutte entre partisans des intérêts privés et partisans de l'intérêt public, représentés notamment par les mouvements populistes, l'idéologie a souvent prévalu sur les faits et les résultats concrets. Même s'il semble s'agir finalement plus d'un concours de circonstances que d'une volonté délibérée, l'action des marchands de Québec a abouti à la mise en place d'un service public, sous la direction de l'État fédéral, au plus grand profit de l'ensemble des milieux économiques canadiens, notamment Montréal, et non pas seulement de Québec. 